\title{
Transgenerational Effects of Maternal Water Condition on the Growth, C:N Stoichiometry and Seed Characteristics of the Desert Annual Atriplex aucheri
}

\author{
Li Jiang ${ }^{1,2,+}$, Zhibin Wen ${ }^{1,2,+} \oplus$, Yunling Zhang ${ }^{3}$, Zhenyong Zhao ${ }^{1,2}$, Mohsin Tanveer ${ }^{4} \oplus$, Changyan Tian ${ }^{1,2}$ \\ and Lei Wang $1,2, *$ (D)
}

1 State Key Laboratory of Desert and Oasis Ecology, Xinjiang Institute of Ecology and Geography, Chinese Academy of Sciences, 818 South Beijing Road, Urumqi 830011, China; jiangli@ms.xjb.ac.cn (L.J.); zhibinwen@ms.xjb.ac.cn (Z.W.); zhaozhy@ms.xjb.ac.cn (Z.Z.); tianchy@ms.xjb.ac.cn (C.T.)

2 University of Chinese Academy of Sciences, Beijing 100049, China

3 General Grassland Station of Xinjiang, Urumqi 830049, China; yunlingzhang2021@163.com

4 Tasmanian Institute of Agriculture, University of Tasmania, Hobart, TAS 7001, Australia; mohsin.tanveer@utas.edu.au

* Correspondence: egiwang@ms.xjb.ac.cn; Tel.: +86-991-782-3189; Fax: +86-991-788-5320

+ These authors contributed equally.

\section{check for} updates

Citation: Jiang, L.; Wen, Z.; Zhang, Y.; Zhao, Z.; Tanveer, M.; Tian, C.; Wang, L. Transgenerational Effects of Maternal Water Condition on the Growth, C:N Stoichiometry and Seed Characteristics of the Desert Annual Atriplex aucheri. Plants 2021, 10, 2362. https: / / doi.org/10.3390/ plants10112362

Academic Editors: Christine Granier and Sophie Brunel-Muguet

Received: 29 September 2021

Accepted: 19 October 2021

Published: 2 November 2021

Publisher's Note: MDPI stays neutral with regard to jurisdictional claims in published maps and institutional affiliations.

Copyright: (c) 2021 by the authors. Licensee MDPI, Basel, Switzerland. This article is an open access article distributed under the terms and conditions of the Creative Commons Attribution (CC BY) license (https:// creativecommons.org/licenses/by/ $4.0 /)$.

\begin{abstract}
Water conditions directly affect plant growth and thus modify reproduction allocation. However, little is known about the transgenerational effects of water conditions on xerophytes. The desert annual Atriplex aucheri produces three types of seeds (A: dormant, ebracteate black seeds; B: dormant, bracteolate black seeds; C: non-dormant, bracteolate brown seeds) on a single plant. The aim of this study was to investigate the effects of low/high water treatment (thereafter progeny water treatment) on aboveground biomass, C:N stoichiometry, and offspring seed characteristics of A. aucheri grown from brown seeds whose mother plants were under low/high water treatment (thereafter maternal water treatment). Progeny water only affected shoot dry weight and seed allocation of type A. Under low progeny water treatment, plants from parents with low maternal water treatment had the lowest biomass. Maternal water did not significantly influence the $\mathrm{C}$ and $\mathrm{N}$ content, however high maternal water increased the $\mathrm{C}: \mathrm{N}$ ratio. Maternal water treatment did not significantly affect seed number. However, plants under low maternal and progeny water treatments had the lowest weight for type B seeds. When progeny plants were under low water treatment, seed allocation of type A, type B, and total seed allocation of plants under high maternal water were significantly lower than those of plants under low maternal water. These results indicate that water conditions during the maternal generation can dramatically contribute to progeny seed variation, but the transgenerational effects depend on the water conditions of progeny plants.
\end{abstract}

Keywords: Atriplex aucheri; seed characteristic; stoichiometry; transgenerational effect; water

\section{Introduction}

Transgenerational effects occur when biotic or abiotic environmental factors act on maternal plants and thereby influence the phenotype of progeny plants. Transmitted factors can be divided into abiotic factors and biotic factors. Abiotic factors include light, nutrient, salinity, water condition, etc. [1-5]. For example, when parental ramets of Centella asiatica were subjected to low light treatment, biomass accumulation and total stolon length of offspring ramets under maternal light conditions were significantly greater than those of offspring ramets under non-maternal light conditions [3]. Biotic factors that have transgenerational effects include competition, herbivory, symbiotic microorganisms, etc. [4,6-8]. For example, when growing in competition, the progeny of parental competition plants of Polygonum persicaria produce great shoot mass and allocate more biomass to the stem than the progeny of parents grown without competition [9]. Most prior studies of the 
transgenerational effects have tested the effects on progeny growth and seed characteristics of plants that produced only one seed type [10-13]. Less is known about the C:N stoichiometry and plants producing heteromorphic seeds [14]. Some evidence indicates that these transgenerational effects might be caused by molecular epigenetic mechanisms [15].

Water stress is one of the most important abiotic factors affecting plant growth and seed yield in arid regions [16-18]. To cope with this stress, plants respond with ecological, physiological, and biochemical changes that affect plant growth and reproduction. Some responses to water stress may be transmitted to subsequent generations. Transgenerational effects of drought on plant growth and progeny seed characteristics have been shown in several studies $[2,13,19]$. For example, the growth differences between barley plants with different stress histories in the maternal generation were more pronounced in progeny grown at drought condition than at optimal water availability [10]. However, the knowledge of the responses of plants with different water demand to drought stress is still incomplete. Moreover, plants respond to stress factors with ecological, physiological, and biochemical changes, and there are few studies regarding transgenerational effects of post-drought descendants.

Most plant species produce one type of seed; however, more than 200 species produce different types of seeds on a single plant [20]. These different seed types generally vary in color, dispersal, dormancy, germination, and even plant growth and reproduction $[20,21]$. Maternal environments can change seed ratio and allocation of heteromorphic seeds [22-24]. For example, plants of Suaeda corniculata subsp. mongolica allocate more biomass to seeds when they are grown in soil of the same salinity as that of their mother and this transgenerational effect on reproductive allocation is also related to maternal seed morph [25]. Thus, the production of heteromorphic seeds reflects not only current environmental conditions but also the maternal environments.

Atriplex aucheri in the family Chenopodiaceae (included in Amaranthaceae) is an early successional annual xerophyte growing in Gobi desert, deserts, and arid valleys [26]. Annual precipitation of these areas is $100-250 \mathrm{~mm}$, but annual potential evaporation is above $2000 \mathrm{~mm}$. This species is native to Xinjiang, China (Afghanistan, Kazakhstan, Turkmenistan; SW Asia, SE Europe). Atriplex aucheri is an optimal organism for studying transgenerational effects of water stress because it is a typical xerophyte and a heterocarpic species producing three types of fruits and seeds on a single plant [27]. Type A seeds are small and black with a 5-lobed perianth. Type B seeds are medium-sized and have a similar appearance to type $\mathrm{A}$, but they are covered by extended bracteoles. Type $\mathrm{C}$ seeds are large and brown and covered by extended bracteoles. Type $C$ seeds of $A$. aucheri are non-dormant and have a high germination percentage, whereas type $A$ and $B$ seeds have non-deep physiological dormancy and low germination percentages [27].

We hypothesized that exposure to water stress of maternal plants of A. aucheri makes progeny plants more resistant to future exposure by stress memory to ensure reproductive success. Specifically, we addressed the following questions: (1) Does maternal water treatment significantly change the $\mathrm{C}$ content, $\mathrm{N}$ content, and C:N ratio? (2) What is the effect of maternal water treatment on the seed production of progeny plants? (3) Do water treatments to maternal plants and progeny plants have an interaction?

\section{Results}

\subsection{Shoot Dry Weight}

A two-way ANOVA showed that shoot dry weights were significantly affected by water treatment experienced by maternal plants and progeny plants (Table 1). When maternal plants were subjected to high water treatment, the shoot dry weight of progeny plants experiencing maternal water environment significantly increased relative to those of progeny plants experiencing low water treatment. Shoot dry weight of progeny plants experiencing low maternal water environment treated with low water had the lowest weight. Under low water treatment, compared with plants with maternal low water treatment, the shoot dry weight of plants with maternal high-water treatment increased $12.8 \%$; under 
high water treatment, compared with plants with maternal low water treatment, the shoot dry weight of plants with high maternal water treatment increased $18.2 \%$ (Figure 1).

Table 1. F values from two-way ANOVA for the effects of progeny water, maternal water, and their interactions on investigated properties of Atriplex aucheri.

\begin{tabular}{cccc}
\hline Source & Progeny Water & Maternal Water & $\begin{array}{c}\text { Progeny Water } \times \\
\text { Maternal Water }\end{array}$ \\
\hline Shoot dry weight $(n=10)$ & $12.94^{* *}$ & $8.47^{*}$ & 0.58 \\
C content $(n=6)$ & 0.48 & 2.95 & 0.26 \\
N content $(n=6)$ & 0.01 & 3.87 & $6.04^{*}$ \\
C: N ratio $(n=6)$ & 0.01 & $5.32^{*}$ & $4.54^{*}$ \\
Seed number of type A $(n=10)$ & 0.12 & 0.75 & 0.15 \\
Seed number of type B $(n=10)$ & 0.94 & 3.10 & 0.38 \\
Seed number of type C $(n=10)$ & 1.31 & 2.61 & 1.48 \\
Total seed number $(n=10)$ & 1.06 & 3.22 & 0.87 \\
Seed weight of type A $(n=50)$ & 0.12 & 0.03 & 1.53 \\
Seed weight of type B $(n=50)$ & 0.06 & $17.62^{* * *}$ & $2.75 *$ \\
Seed weight of type C $(n=50)$ & 1.73 & 0.37 & 0.85 \\
Seed yield of type A $(n=10)$ & 0.97 & 0.53 & 0.72 \\
Seed yield of type B $(n=10)$ & 0.22 & $5.56^{*}$ & 1.50 \\
Seed yield of type C $(n=10)$ & 0.97 & 1.70 & 1.82 \\
Total seed yield $(n=10)$ & 0.60 & 2.70 & 0.24 \\
Seed allocation of type A $(n=10)$ & $7.93^{* *}$ & $4.49^{*}$ & 0.95 \\
Seed allocation of type B $(n=10)$ & 0.19 & $10.28^{* *}$ & 2.73 \\
Seed allocation of type C $(n=10)$ & 0.15 & 1.39 & 2.20 \\
Total seed allocation $(n=10)$ & 0.92 & $7.23^{*}$ & \\
\hline
\end{tabular}

Note: ${ }^{*} p<0.05,{ }^{* *} p<0.01,{ }^{* * *} p<0.001$.

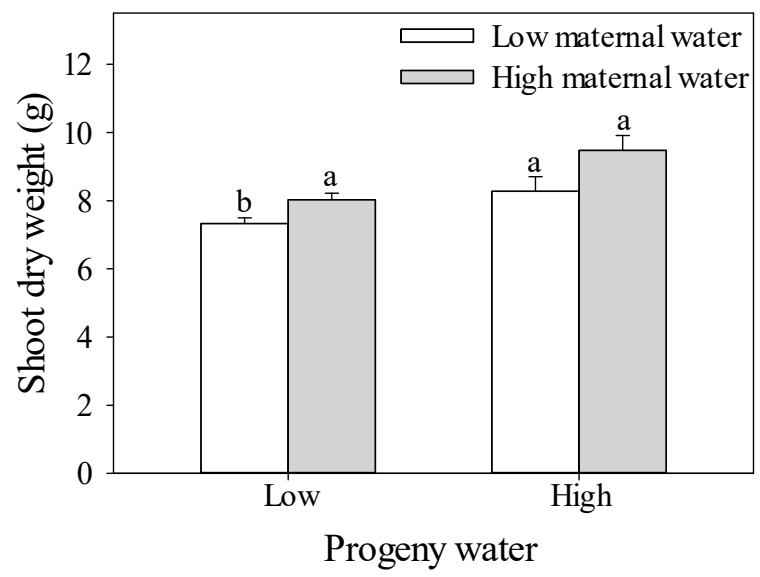

Figure 1. Effects of maternal and progeny water treatments on shoot dry weight per plant. Different lowercase letters represent significantly different means between plants with different maternal water treatments under the same progeny water treatment according to $t$-test $(p<0.05)$.

\subsection{Content, $N$ Content and C:N Ratio}

$\mathrm{C}$ content was not significantly affected by water treatment for maternal plants and progeny plants and their interaction (Table 1). C content ranged from $377.44 \mathrm{mg} \mathrm{kg}^{-1}$ to $386.57 \mathrm{mg} \mathrm{kg}^{-1}$ (Figure 2A). 


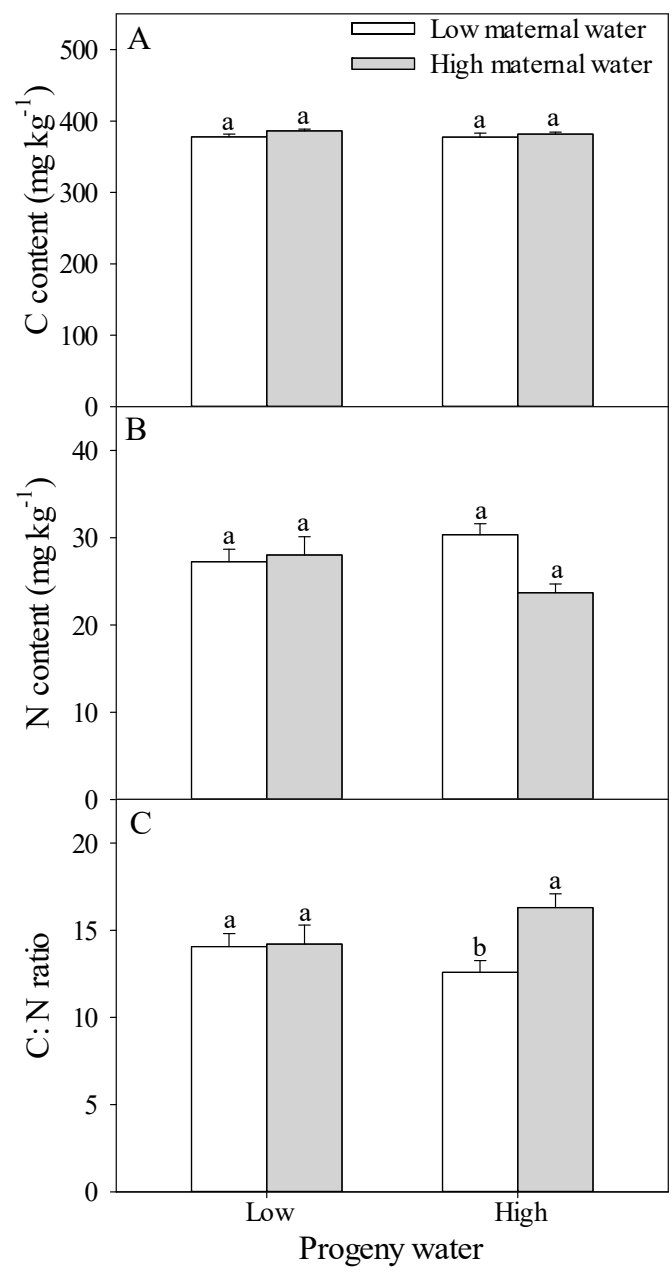

Figure 2. Effects of maternal and progeny water treatments on (A) C content, (B) N content, and (C) $\mathrm{C}: \mathrm{N}$ ratio. Different lowercase letters represent significantly different means between plants with different maternal water treatments under the same progeny water treatment according to $t$-test $(p<0.05)$.

$\mathrm{N}$ content was significantly affected by the interaction of water treatments for maternal plants and progeny plants, but not by the water treatment for maternal plants or water treatment for progeny plants (Table 1). The lowest value $\left(23.68 \mathrm{mg} \mathrm{kg}^{-1}\right)$ for $\mathrm{N}$ content was from the progeny plants with high water treatment grown from seeds on maternal plants with high water treatment (Figure 2B).

$\mathrm{C}: \mathrm{N}$ ratio was significantly affected by the water treatment for maternal plants and the interaction between water treatments for maternal plants and for progeny plants, but not affected by water treatment for progeny plants (Table 1). The highest value (16.30) for $\mathrm{C}: \mathrm{N}$ ratio was from the progeny plants with high water treatment grown from seeds on maternal plants with high water treatment (Figure 2C).

\subsection{Seed Traits}

Seed numbers of type A, type B, type C and total seed number were not significantly affected by water treatment for maternal plants and for progeny plants, and their interaction (Table 1 and Figure 3). When progeny plants were under low water treatment, compared with plants under low maternal water, seed number of type $C$, and total seed number of plants under high maternal water decreased $66.54 \%$ and $23.93 \%$, respectively (Figure 4C,D). 


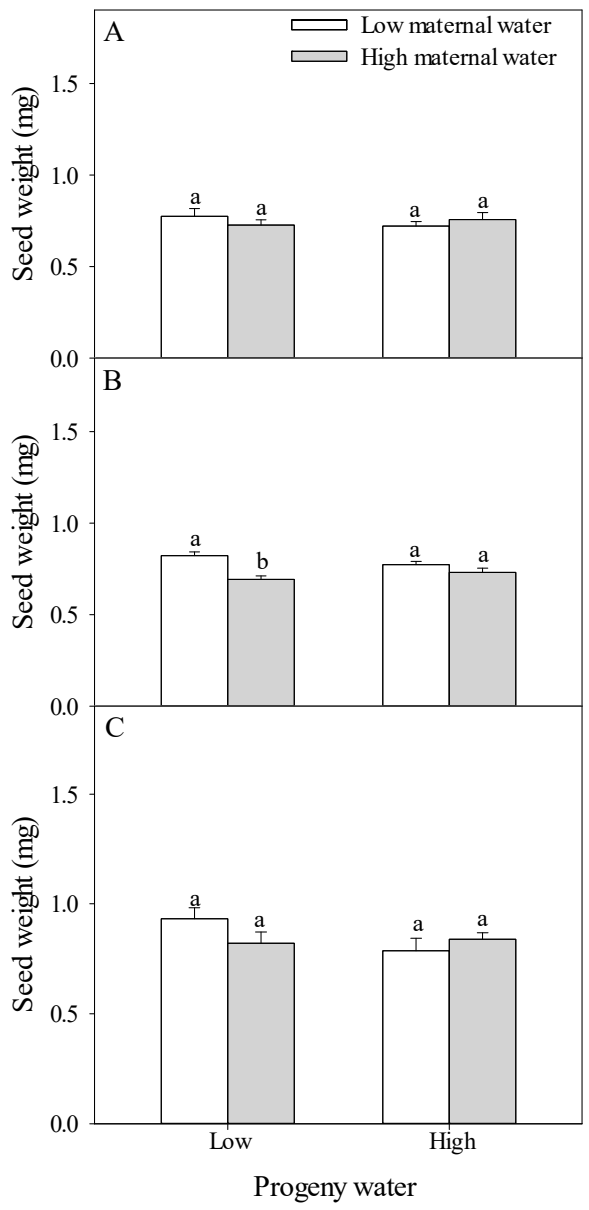

Figure 3. Effects of maternal and progeny water treatments on (A) seed weight of type A, (B) seed weight of type B, and (C) seed weight of type C. Different lowercase letters represent significantly different means between plants with different maternal water treatments under the same progeny water treatment according to $t$-test $(p<0.05)$.

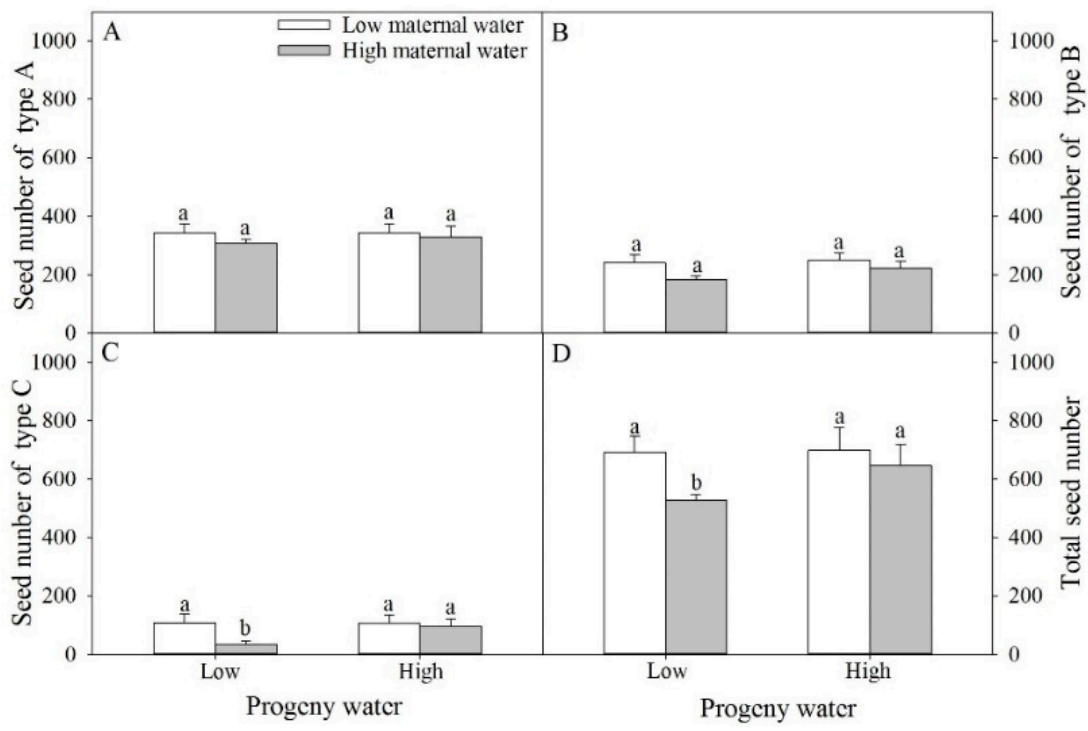

Figure 4. Effects of maternal and progeny water treatments on (A) seed number of type A, (B) seed number of type B, (C) seed number of type C, and (D) total seed number per plant. Different lowercase letters represent significantly different means between plants with different maternal water treatments under the same progeny water treatment according to $t$-test $(p<0.05)$. 
Seed weights of type A and type $C$ were not significantly affected by water treatment for maternal plants and for progeny plants, and their interaction, but seed weight of type $B$ was significantly affected by water treatment for maternal plants, and their interaction (Table 1). When progeny plants were under low water treatment, compared with plants under high maternal water, seed weight of type B of plants under low maternal water increased $18.70 \%$ (Figure 3B).

Seed yield of type A, type C, and total seed yield were not significantly affected by water treatment for maternal plants and for progeny plants and their interaction (Table 1 and Figure 5). Seed yield of type B was significantly affected by water treatment for maternal plants (Table 1 and Figure 5B). When progeny plants were under low water treatment, seed yield of type $B$ and total seed number of plants under high maternal water were significantly lower than that of plants under low maternal water (Figure 5B,D).

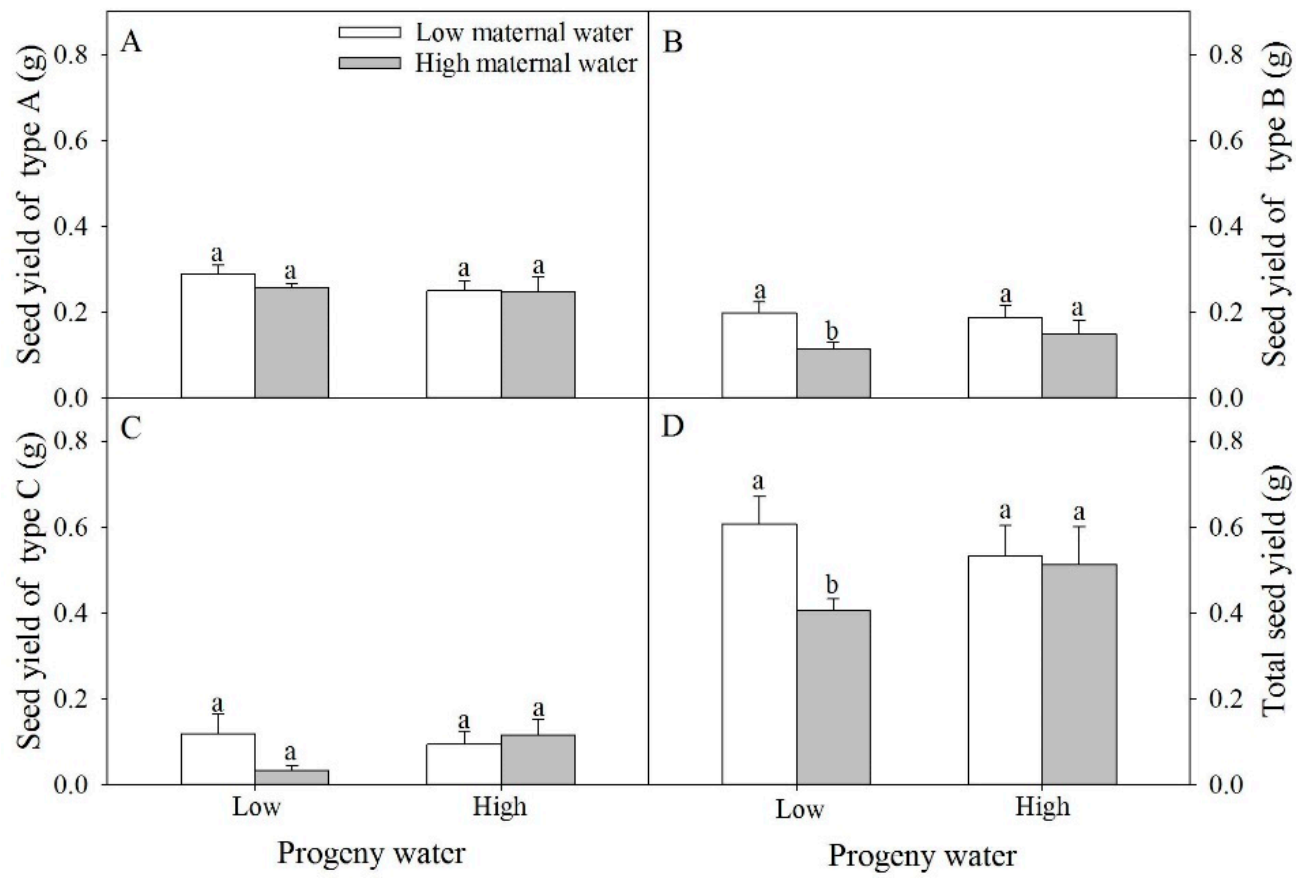

Figure 5. Effects of maternal and progeny water treatments on (A) seed yield of type A, (B) seed yield of type B, (C) seed yield of type C, and (D) total seed yield per plant. Different lowercase letters represent significantly different means between plants with different maternal water treatments under the same progeny water treatment according to $t$-test $(p<0.05)$.

Seed allocation of type A was significantly affected by water for maternal plants and for progeny plants (Table 1). Seed allocations of type B and total seed allocation were significantly affected by water for maternal plants. Seed allocation of type C was not significantly affected by water for maternal plants and for progeny plants and their interaction. When progeny plants under low water treatment, seed allocation of type A, type $B$ and total seed allocation of plants under high maternal water were significantly lower than that of plants under low maternal water (Figure 6A,B,D). 


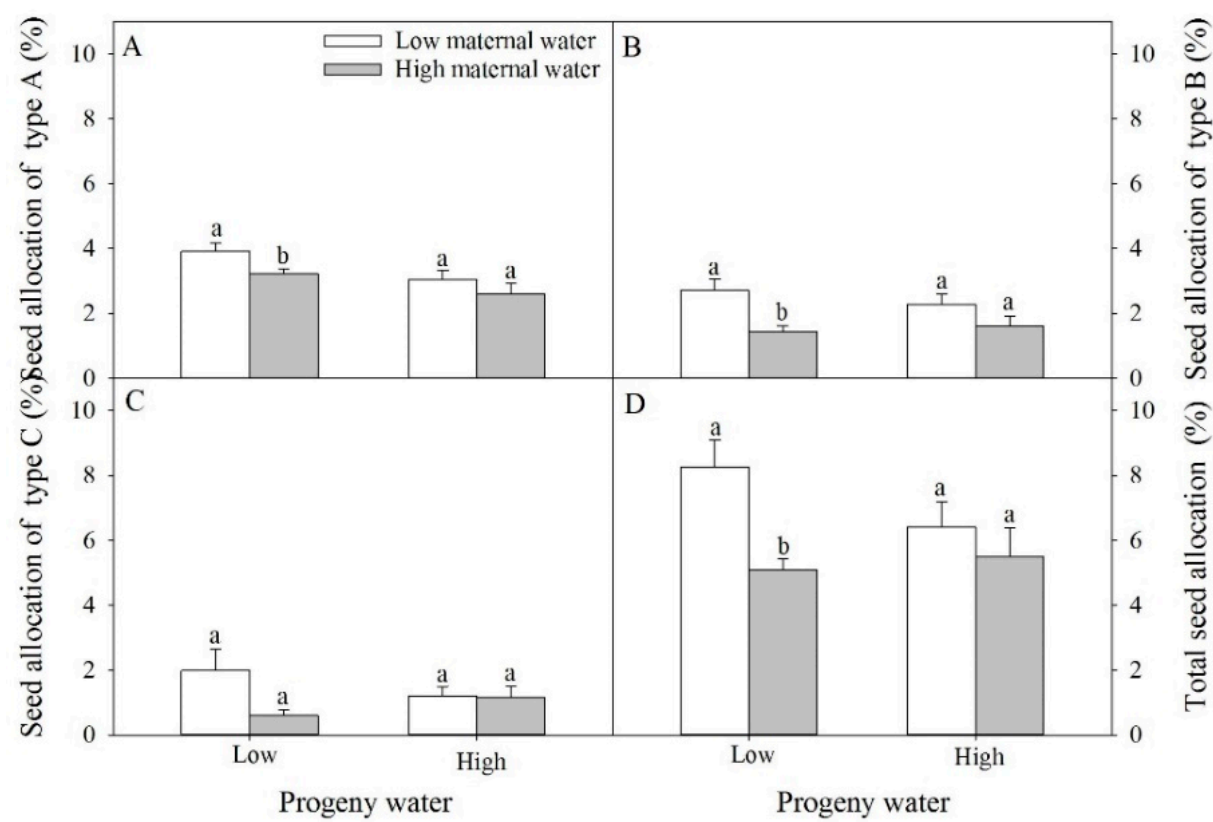

Figure 6. Effects of maternal and progeny water treatments on (A) seed allocation of type A, (B) seed allocation of type B, (C) seed allocation of type C, and (D) total seed allocation. Different lowercase letters represent significantly different means between plants with different maternal water treatments under the same progeny water treatment according to $t$-test $(p<0.05)$.

\section{Discussion}

Although seed germination ecology, plant growth, and utilization of xerophytes, for example, A. aucheri, have been studied extensively [28-30], these data are the first addressing transgenerational effects of low/high water treatment on C:N stoichiometry and progeny seed characteristics of xerophytes. In addition, our data indicated that though the maternal water treatment significantly influences shoot dry weight and C:N ratio, though the treatment does not significantly affect $\mathrm{C}$ and $\mathrm{N}$ contents. Maternal water treatment affects seed weight and seed yield of type B of progeny plants. Water treatments to maternal plants and progeny plants have an interaction on $\mathrm{N}$ content, $\mathrm{C}: \mathrm{N}$ ratio, and seed weight of type $B$. The results indicated that exposure to water stress of maternal plants of $A$. aucheri makes progeny plants more resistant to future exposure to ensure reproductive success.

Transgenerational effects of drought stress have been well documented [2,10,31]. However, there are few studies about transmitted developmental effects on xerophytes. We found that maternal water conditions induced significant effects on progeny growth, C:N ratio, and seed allocation. Moreover, the drought stress imposed transgenerational effects on progeny growth and reproduction that were distinct from the mesophytes $[2,12,31]$. Though researches of other plant types are needed to test the transgenerational effects, such effects might be important in xerophytes, which are characterized by extreme drought conditions [32]. Our study provided new insight into transgenerational effects of water treatment by demonstrating substantial developmental effects on $\mathrm{C}: \mathrm{N}$ ratio and seed allocation of different seed types.

Variation in biomass allocation to vegetative and reproductive parts is a crucial life history strategy for annuals in response to the changing environmental conditions, especially for frequently disturbed and environment extreme habitats [14,25]. Our results showed that both maternal water condition and progeny water condition could significantly influence the aboveground biomass of progeny plants of A. aucheri. This indicates that even for the xerophytes, a high level of maternal and progeny water is beneficial for the growth of progeny plants. However, the increase of biomass did not result in the increase of seed number and seed yield of different seed types. Contrarily, when progeny plants were 
cultured under low water treatment, seed yield was higher when the maternal plants were cultured under low water treatment. Considering the habitat of this xerophyte is deserts and arid valleys, the transgenerational effects of drought for progeny in similar conditions might be a kind of reproductive assurance.

Previous studies showed that plants generally have higher $\mathrm{C}: \mathrm{N}$ ratio under drought stress [33]. Our results showed that C:N ratio of progeny plants were low and not significantly affected by progeny water treatment, but by the maternal water treatment. The low C:N ratio was also found in other plants grown in arid land [34]. Under high progeny water treatment, plants treated with low maternal water had lower $C: N$ ratio than plants treated with high maternal water. The lower C:N ratio in progeny of low water-treated mothers may be due to shoots containing more metabolic, N-rich tissue. This might be an adaptation to cope with unusual high-water condition in arid land.

The seed traits of $A$. aucheri showed different responses to water treatment for maternal plants and for progeny plants. Our results indicated that water conditions had transgenerational effects on seed weight and seed yield of type B, seed allocation of type $A$ and type $B$, and total seed allocation. When progeny plants were under low water treatment, seed allocation of type A, type B, and total seed allocation of plants under low maternal water were significantly higher than those of plants under high maternal water. Thus, transgenerational effects on offspring seed traits varied depending on maternal and progeny water environments. Plants generally allocate a certain proportion of resources to seeds. Thus, there is a trade-off between seed number and seed weight [35]. Our results support this optimization model as different maternal and progeny water treatments had no effect on seed number and seed weight, except the seed weight of type B. Under low progeny water treatment, plants grown from seeds with low maternal water treatment had significantly higher seed allocation, especially for type A and type B. The results indicate that under drought stress, maternal fitness of $A$. aucheri is increased by increasing offspring fitness.

\section{Conclusions}

In conclusion, this study showed that the maternal water condition can induce transgenerational effects on offspring seed allocation of seed-heteromorphic xerophyte. The allocation depends on water conditions in both maternal and offspring generations. Maternal low water conditions conferred a seed allocation advantage when offspring plants were under low water conditions. Interestingly, the transgenerational effects on offspring seed characteristics of different types were different. Importantly, for most measured indexes, there was no interaction between maternal water and progeny water treatments, suggesting both water treatments influenced the progeny in different ways. Further research should pay more attention to the transgenerational effects among multiple generations and the progeny grown from different types of seeds.

\section{Materials and Methods}

\subsection{Seed Collection}

Seeds (F1) of A. aucheri sowed in this experiment was collected from a previous pot experiment carried out from early April 2011 to early October 2011. The experimental design constituted low and high-water treatments (Figure 7). The experimental location and methods were the same as the current pot experiment. Thus, the description for previous pot experiment was given in the methods of current experiment. After removing the bracteoles, seeds were sorted according to the water treatment and seed types, and then stored in paper bags at room temperature. Since type $C$ seeds are non-dormant and have the highest germination percentages (usually up to 95\%; [27]), they were used in the current pot experiment (Figure 1). 

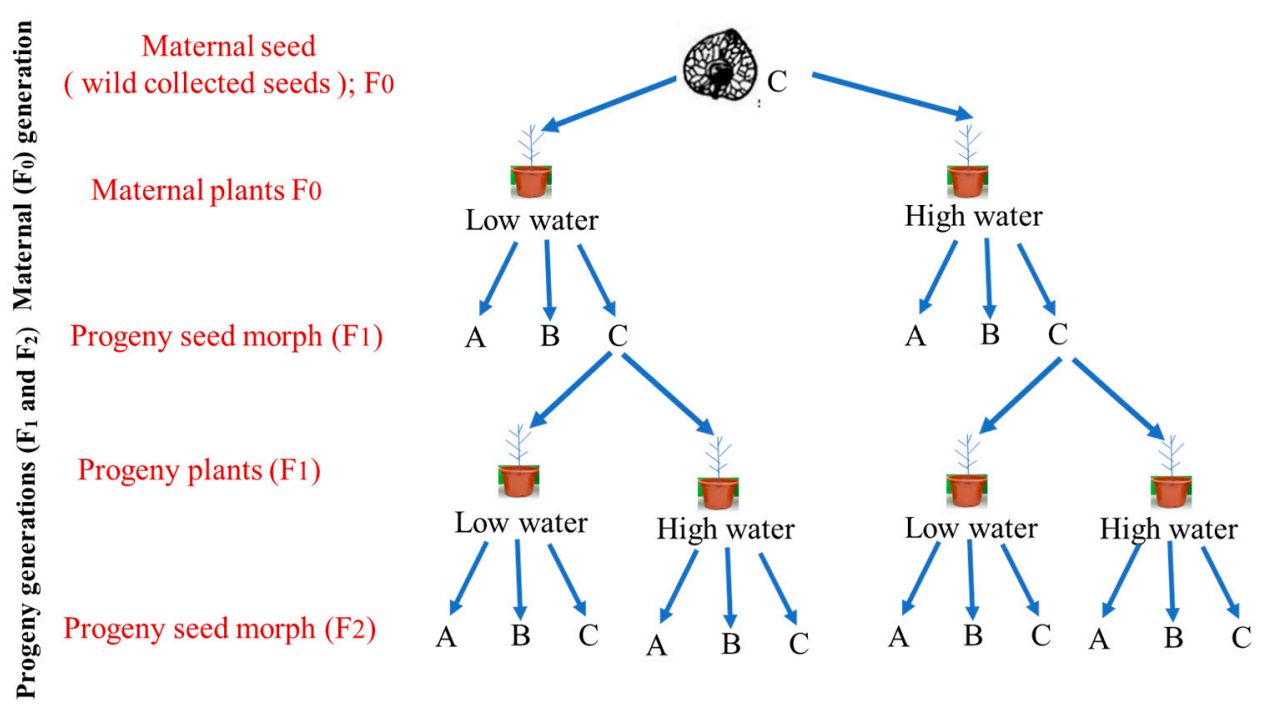

Figure 7. Experimental design for plants and seeds of maternal generation and progeny generations. A, B, and C indicate the seeds of type A, type B, and type C, respectively.

Seeds $\left(\mathrm{F}_{0}\right)$ of $A$. aucher sowed in previous pot experiment were collected from a natural population growing in the hilly desert on the southern edge of Junggar Basin in Xinjiang in October 2010. Seeds were sorted according to seed types and only type C seeds were used in the previous pot experiment.

\subsection{Pot Experiment}

About 20 type $\mathrm{C}$ seeds $\left(\mathrm{F}_{1}\right)$ from low and high-water treatments were sown at $2 \mathrm{~mm}$ soil depth into individual plastic pots $(17 \mathrm{~cm}$ deep and $16 \mathrm{~cm}$ in diameter) filled with $2 \mathrm{~L}$ loam soil and vermiculite mixture $(3: 1 \mathrm{v} / \mathrm{v})$ on 15 April 2012. For fertilization, a commonly available granular lawn fertilizer (Osmocote 301, Scotts, Marysville, OH, USA, 15N: 11P: 13K: $2 \mathrm{Mg}$ ) and a commercial nutrient solution (Peters1, Scotts, Marysville, OH, USA, 20N: 20P: 20K) were used. Each pot received $6 \mathrm{~g}$ Osmocote 301 once before sowing as basic fertilizer. The seedlings were thinned to one per pot, 20 days after sowing. To reduce variation in initial seedling size, only seedlings of a similar height for each seed type were kept. For water treatment, each pot received $100 \mathrm{~mL}$ water (low) and $200 \mathrm{~mL}$ water (high) per day. A randomized block design with ten replicates was used. Each block contained 4 pots representing a combination of two water treatments for maternal plants and two treatments for progeny plants. The experiment was terminated on 14 October 2012, and then the shoot and progeny seeds $\left(\mathrm{F}_{2}\right)$ were harvested.

\subsection{Shoot Dry Weight}

After harvesting, shoot dry weight was recorded after oven drying at $60{ }^{\circ} \mathrm{C}$ until constant weight was obtained.

\subsection{Content, N Content and C:N Ratio}

Before chemical analysis, the dried shoot materials were ground in a ball mill, and then passed through a $0.25 \mathrm{~mm}$ sieve. The ball mill was cleaned completely after each sample milling. The shoot $\mathrm{C}$ content and $\mathrm{N}$ content were determined using an elemental analyzer (FLASHEA 1112 Series CNS Analyzer, Thermo, Waltham, MA, USA).

\subsection{Seed Traits}

The number of seeds for different seed types on a single plant was counted after detached and cleaned. Seed weight of 5 randomly selected seeds for each morph from each plant were measured by using electronic balance (Precision $=0.00001 \mathrm{~g}$; Sartorius, SQP). Seed yield of different seed types for each individual plant was measured by using 
electronic balance (Precision $=0.0001 \mathrm{~g}$; Chyo Balance, JPN-200W). Seed allocation $(\%)=$ (seed yield/shoot dry weight) $\times 100 \%$.

\subsection{Statistical Analyses}

Statistical analysis was performed using the SPSS software (version 16.0). All data were expressed as mean \pm standard error (S.E). Two-way ANOVA was used to determine the significant effects of water treatment for progeny plants and for maternal plants, and their interaction on shoot dry weight, $\mathrm{C}$ content, $\mathrm{N}$ content, $\mathrm{C}: \mathrm{N}$ ratio, seed number, seed weight, seed yield, and seed allocation.

Author Contributions: Conceptualization, L.W., Z.W., M.T. and L.J.; investigation, Z.W. and L.J.; writing-original draft preparation, Z.W., L.J. and L.W.; writing-review and editing, M.T., C.T., Y.Z., Z.Z. and L.W.; visualization, Y.Z. and L.W.; project administration, L.W.; funding acquisition, Z.Z. All authors have read and agreed to the published version of the manuscript.

Funding: This work was supported by the Special Project for the Innovation Environment (Talents and Bases) Construction in Xinjiang Uygur Autonomous Region-Resource Sharing Platform Construction Project (PT2033) and West Light Talent Program of the Chinese Academy of Sciences (2019-YDYLTD-001).

Institutional Review Board Statement: Not applicable.

Informed Consent Statement: Not applicable.

Data Availability Statement: Data is contained within the article.

Conflicts of Interest: The authors declare no conflict of interest.

\section{References}

1. Cano, L.; Fuertes-Mendizabal, T.; Garcia-Baquero, G.; Herrera, M.; Gonzalez-Moro, M.B. Plasticity to salinity and transgenerational effects in the nonnative shrub Baccharis halimifolia: Insights into an estuarine invasion. Am. J. Bot. 2016, 103, 808-820. [CrossRef]

2. Hatzig, S.V.; Nuppenau, J.N.; Snowdon, R.J.; Schiessl, S.V. Drought stress has transgenerational effects on seeds and seedlings in winter oilseed rape (Brassica napus L.). BMC Plant Biol. 2018, 18, 297. [CrossRef]

3. Li, K.-N.; Chen, J.-S.; Wei, Q.; Li, Q.; Lei, N.-F. Effects of transgenerational plasticity on morphological and physiological properties of stoloniferous herb Centella asiatica subjected to high/low light. Front. Plant Sci. 2018, 9, 1640. [CrossRef]

4. Dong, B.-C.; Alpert, P.; Yu, F.-H. Transgenerational effects of herbivory and soil nutrients transmitted via vegetative reproduction in the clonal plant Alternanthera philoxeroides. Perspect. Plant Ecol. Evol. Sys. 2019, 41, 125498. [CrossRef]

5. Lazaro-Lobo, A.; Herrera, M.; Campos, J.A.; Cano, L.; Goni, E.; Ervin, G.N. Influence of local adaptations, transgenerational effects and changes in offspring's saline environment on Baccharis halimifolia L. under different salinity and light levels. Environ. Exp. Bot. 2020, 177, 104134. [CrossRef]

6. Agrawal, A.A. Transgenerational consequences of plant responses to herbivory: An adaptive maternal effect? Am. Nat. 2001, 157, 555. [CrossRef] [PubMed]

7. Gundel, P.E.; Rudgers, J.A.; Whitney, K.D. Vertically transmitted symbionts as mechanisms of transgenerational effects. Am. J. Bot. 2017, 104, 787-792. [CrossRef] [PubMed]

8. Rottstock, T.; Kummer, V.; Fischer, M.; Joshi, J. Rapid transgenerational effects in Knautia arvensis in response to plant community diversity. J. Ecol. 2017, 105, 714-725. [CrossRef]

9. Waterman, R.; Sultan, S.E. Transgenerational effects of parent plant competition on offspring development in contrasting conditions. Ecology 2021, e03531. [CrossRef]

10. Nosalewicz, A.; Siecinska, J.; Smiech, M.; Nosalewicz, M.; Wiacek, D.; Pecio, A.; Wach, D. Transgenerational effects of temporal drought stress on spring barley morphology and functioning. Environ. Exp. Bot. 2016, 131, 120-127. [CrossRef]

11. González, A.P.R.; Dumalasová, V.; Rosenthal, J.; Skuhrovec, J.; Latzel, V. The role of transgenerational effects in adaptation of clonal offspring of white clover (Trifolium repens) to drought and herbivory. Evol. Ecol. 2017, 31, 345-361. [CrossRef]

12. Yin, J.-J.; Zhou, M.; Lin, Z.; Lin, Z.-R.; Li, Q.-S.; Zhang, Y.-Y. Transgenerational effects benefit offspring across diverse environments: A meta-analysis in plants and animals. Ecol. Lett. 2019, 22, 1976-1986. [CrossRef] [PubMed]

13. Liu, H.; Able, A.J.; Able, J.A. Transgenerational effects of water-deficit and heat stress on germination and seedling vigour-new insights from durum wheat microRNAs. Plants 2020, 9, 189. [CrossRef] [PubMed]

14. Walter, J.; Harter, D.E.V.; Beierkuhnlein, C.; Jentsch, A. Transgenerational effects of extreme weather: Perennial plant offspring show modified germination, growth and stoichiometry. J. Ecol. 2016, 104, 1032-1040. [CrossRef]

15. Adrian-Kalchhauser, I.; Sultan, S.E.; Shama, L.N.S.; Spence-Jones, H.; Tiso, S.; Valsecchi, C.I.K.; Weissing, F.J. Understanding 'Non-genetic' Inheritance: Insights from molecular evolutionary crosstalk. Trends Ecol. Evol. 2020, 35, 1078-1089. [CrossRef] [PubMed] 
16. Merino-Martin, L.; Courtauld, C.; Commander, L.; Turner, S.; Lewandrowski, W.; Stevens, J. Interactions between seed functional traits and burial depth regulate germination and seedling emergence under water stress in species from semi-arid environments. J. Arid Environ. 2017, 147, 25-33. [CrossRef]

17. Duncan, C.; Schultz, N.L.; Good, M.K.; Lewandrowski, W.; Cook, S. The risk-takers and -avoiders: Germination sensitivity to water stress in an arid zone with unpredictable rainfall. AoB Plants 2019, 11, plz066. [CrossRef]

18. Lewandrowski, W.; Stevens, J.C.; Webber, B.L.; Dalziell, E.L.; Trudgen, M.S.; Bateman, A.M.; Erickson, T.E. Global change impacts on arid zone ecosystems: Seedling establishment processes are threatened by temperature and water stress. Ecol. Evol. 2021, 11, 8071-8084. [CrossRef]

19. Zheng, X.; Chen, L.; Li, M.; Lou, Q.; Luo, L. Transgenerational variations in DNA methylation induced by drought stress in two rice varieties with distinguished difference to drought resistance. PLoS ONE 2013, 8, e80253. [CrossRef]

20. Imbert, E. Ecological consequences and ontogeny of seed heteromorphism. Perspect Plant Ecol. Evol. Sys. 2002, 5, 13-36. [CrossRef]

21. Mandák, B. Seed heteromorphism and the life cycle of plants: A literature review. Preslia 1997, 69, 129-159.

22. Cheplick, G.P.; Quinn, J.A. The shift in aerial/subterranean fruit ratio in Amphicarpum purshii: Causes and significance. Oecologia 1983, 57, 374-379. [CrossRef] [PubMed]

23. Sadeh, A.; Guterman, H.; Gersani, M.; Ovadia, O. Plastic bet-hedging in an amphicarpic annual: An integrated strategy under variable conditions. Evol. Ecol. 2009, 23, 373-388. [CrossRef]

24. Lu, J.-J.; Tan, D.-Y.; Baskin, J.M.; Baskin, C.C. Germination season and watering regime, but not seed morph, affect life history traits in a cold desert diaspore-heteromorphic annual. PLoS ONE 2014, 9, e102018. [CrossRef]

25. Yang, F.; Yang, X.-J.; Baskin, J.M.; Baskin, C.C.; Cao, D.-C.; Huang, Z.-Y. Transgenerational plasticity provides ecological diversity for a seed heteromorphic species in response to environmental heterogeneity. Perspect. Plant Ecol. Evol. Sys. 2015, 17, 201-208. [CrossRef]

26. Flora of China Editorial Committee. Flora of China; Science Press and Missouri Botanical Garden Press: Beijing, China, 2003; Volume 5.

27. Wang, L.; Wang, H.-L.; Zhang, K.; Tian, C.-Y. Effects of maternal nutrient and water availability on seed production, size and germination of heterocarpic Atriplex aucheri. Seed Sci. Technol. 2015, 43, 71-79. [CrossRef]

28. Huang, J.; Tian, C.-Y.; Bian, W.-G.; Yin, H.-L.; Ren, J.; Chen, C.-X. Response of growth of four halophyte species in oil-contaminated soil. Arid Zone Res. 2014, 31, 100-104. (In Chinese with English Abstract)

29. Wei, Y.; Liu, P.-W.; An, S.-Z. Study on fruit polymorphism and germination measures of Atriplex aucheri Moq. Seeds. Arid Zone Res. 2007, 24, 835-839. (In Chinese with English Abstract)

30. Wang, W.-H.; Jiang, L. Effects of four kinds of sodium salinity stress on seed germination and young seedling growth of Atriplex aucheri. Chin. J. Grassland 2020, 42, 23-29. (In Chinese with English Abstract)

31. Chathurika, W.; Raja, R.K.; Jason, K.L.; Wei, G.; Nacer, B. Drought stress has transgenerational effects on soybean seed germination and seedling vigor. PLoS ONE 2019, 14, e0214977.

32. Pias, B.; Matesanz, S.; Herrero, A.; Gimeno, T.E.; Escudero, A.; Valladares, F. Transgenerational effects of three global change drivers on an endemic Mediterranean plant. Oikos 2010, 119, 1435-1444. [CrossRef]

33. Sardans, J.; Penuelas, J.; Estiarte, M.; Prieto, P. Warming and drought alter C and N concentration, allocation and accumulation in a Mediterranean shrubland. Glob. Chang. Biol. 2008, 14, 2304-2316. [CrossRef]

34. Wang, L.-L.; Zhao, G.-X.; Li, M.; Zhang, M.-T.; Zhang, L.-F.; Zhang, X.-F.; An, L.-Z.; Xu, S.-J. C:N:P stoichiometry and leaf traits of halophytes in an arid saline environment, Northwest China. PLoS ONE 2015, 10, e0119935. [CrossRef] [PubMed]

35. Fenner, M.; Thompson, K. The Ecology of Seeds; Cambridge University Press: Cambridge, UK, 2005. 